\title{
Development of an experimental set-up for the measurement of neutron-induced fission and capture cross sections of radioactive fissile nuclei
}

\author{
I. Companis ${ }^{1,5, a}$, M. Aïche ${ }^{1}$, L. Mathieu ${ }^{1}$, P. Schillebeeckx ${ }^{2}$, J. Heyse ${ }^{2}$, G. Barreau ${ }^{1}$, S. Czajkowski ${ }^{1}$, Q. \\ Ducasse $^{1}$, F. Gunsing 3 , B. Jurado ${ }^{1}$, G. Kessedjian ${ }^{4}$, J. Matarranz ${ }^{1}$, A.J.M. Plompen ${ }^{2}$, \\ and I. Tsekhanovich ${ }^{1}$ \\ ${ }^{1}$ Centre d'Etudes Nucléaires Bordeaux Gradignan, CNRS/IN2P3, Univ. Bordeaux 1, \\ Chemin du Solarium, 33175 Gradignan, France \\ ${ }^{2}$ EC-JRC, Institute for Reference Materials and Measurements, Reteiseweg 111, 2440 Geel, Belgium \\ ${ }^{3}$ CEA/Saclay - DSM/DAPNIA/SPhN, 91191 Gif-sur-Yvette, France \\ ${ }^{4}$ Laboratoire de Physique Subatomique et de Cosmologie, CNRS/IN2P3, Univ. Joseph Fourier, INPG, \\ 53 avenue des Martyrs, 38026 Grenoble Cedex, France \\ ${ }^{5}$ Horia-Hulubei National Institute for Physics and Nuclear Engineering, PO Box MG-6, \\ 077125 Bucharest-Magurele, Romania
}

\begin{abstract}
A new experimental set-up for a simultaneous measurement of neutron induced capture and fission cross sections was designed, assembled and optimized. The measurements will be performed at GEel LINear Accelerator (GELINA) neutron timeof-flight facility in Belgium, where neutron cross sections can be measured over a wide energy range with high energy resolution. The fission events detector consists of a dedicated multi-plate high efficiency fission ionization chamber (IC). The $\gamma$-rays produced in capture reaction are detected by an efficient array of $\mathrm{C}_{6} \mathrm{D}_{6}$ scintillators. Fission $\gamma$-rays events are distinguished from capture events by the anticoincidence signals from the IC and the $\mathrm{C}_{6} \mathrm{D}_{6}$ detectors. For the undetected fission events a correction has to be applied with respect to the efficiency of the IC that should be high and known with a high precision. Another important issue is the good separation between fission-fragment (FF) and the high alpha pile-up. The performances of the IC during test experiments are presented, focusing in particular on the detection efficiency.
\end{abstract}

\section{Introduction}

The ${ }^{232} \mathrm{Th} /{ }^{233} \mathrm{U}$ fuel cycle is an alternative to the actual ${ }^{238} \mathrm{U} /{ }^{239} \mathrm{Pu}$ fuel cycle, with the main advantage of a substantially lower production of highly radiotoxic nuclear waste. The ${ }^{232} \mathrm{Th}$ cycle, with its lower atomic and mass numbers, produces a reduced amount of minor actinides, especially americium and curium. Little used industrially, the thorium cycle has also been less explored. Therefore, for the

\footnotetext{
ae-mail: companis@cenbg.in2p3.fr
}

This is an Open Access article distributed under the terms of the Creative Commons Attribution License 2.0, which permits unrestricted use, distribution, and reproduction in any medium, provided the original work is properly cited. 
feasibility study and design of innovative systems based on the use of ${ }^{232} \mathrm{Th}$ cycle, accurate and consistent neutron cross section data are required. Recent uncertainty analysis of the breeding of the thorium cycle $[1,2]$ highlighted severe requirements for the accuracy of the neutron induced fission and capture cross sections of ${ }^{233} \mathrm{U}$, especially in the Resolved Resonance Region (RRR).

The criticality analysis and design calculation for thorium-based reactors require an accurate knowledge of the neutron induced cross sections of ${ }^{233} \mathrm{U} /{ }^{239} \mathrm{Pu}$, since this isotope plays the same role as

${ }^{235} \mathrm{U}$ in standard nuclear power plants. Neutron capture cross sections measurements on many actinides are complicated by the difficulty in separating capture $\gamma$-rays from the large fission fragment $\gamma$-ray decay. Also, the few existing cross section measurements are quite old and may be subject to systematic errors: data from M. J. Cabell [3] in the thermal energy, from F.D. Brooks [4] and especially L. W. Weston [5] in the RRR region, and from J. C. Hopkins [6] in the fast region. Therefore, new and accurate measurements for $\sigma(\mathrm{n}, \mathrm{f})$ and $\sigma(\mathrm{n}, \gamma)$ of ${ }^{233} \mathrm{U}$ are planned on different facilities. Recent measurements for these reactions have been performed by the $\mathrm{n}$-TOF collaboration at CERN and preliminary results were published [7]. They concluded that the capture cross section data of Weston [5] are overestimated by up to $20 \%$ in the RRR region. Therefore, these results can than be considered to be poorly known and new measurements are needed. In this respect, the ACEN group of CENBG decided to focus its interest on these reactions together with IRMM Geel (Belgium) [8] where the final experiment will be carried out on the neutron time-of-flight GELINA facility.

\section{Experimental procedure}

Measurements of ${ }^{233} \mathrm{U}$ capture cross sections are complicated by $\gamma$-ray background originating from neutron-induced fission reaction. Typically, fission fragment detectors are used to identify the neutroninduced fission reaction events and to separate them during the analysis from those of capture reaction. This requires that ${ }^{233} \mathrm{U}$ samples to be thin enough to achieve a high fission fragment detection efficiency. On the other hand, the thin sample diminishes the counting statistics of the measurement. As a result, the residual spectrum remains contaminated with both prompt fission $\gamma$-rays and delayed $\gamma$-rays that follow $\beta$ decay of fission products. In addition, the neutron scattering background and the background associated with the neutron beam facility has to be subtracted. The removal of several background components leads to large uncertainties and, if not performed accurately, the results will present systematic errors.

As for ${ }^{233} \mathrm{U} \sigma(\mathrm{n}, \mathrm{f})$ is 5 to 10 times greater than its $\sigma(\mathrm{n}, \gamma)$, and as prompt fission $\gamma$ multiplicity is nearly twice the capture $\gamma$ multiplicity, there are 10 to 15 times more $\gamma$-rays coming from fission in comparison with those coming from a capture reaction. The discrimination between these $\gamma$-rays is one of the challenging issues in this experiment. In this work we have chosen the VETO method: the $\gamma$-rays detected in coincidence with the FF correspond to a fission event and, the capture event is identified by putting a VETO on FF. A schematic view of the experimental set-up is displayed in Figure 1a and a representative diagram of the detection events is shown in Figure $1 \mathrm{~b}$.

The fission efficiency can be measured via the prompt neutrons emitted by FF. These neutrons are detected in the liquid scintillators, and can be disentangled from $\gamma$-rays through Pulse Shape Discrimination method. The IC afficiency is then given by the ratio between the numbers of detected neutrons in coincidence with the fission fragments and the total number of detected neutrons.

To achieve this performance the efficiency of the IC has to be known very precisely. Since some of the FF are affected by backscattering and self-absorption, the fission efficiency is not $100 \%$, implying that some fission events remain undetected. If a fission event is not detected in IC, one of the six $\mathrm{C}_{6} \mathrm{D}_{6}$ scintillators could detect a prompt $\gamma$-ray and the fission event will be interpreted as a capture event. As there are much more fission $\gamma$-rays than capture $\gamma$-rays, the uncertainty on fission eficiency will lead to an increased uncertainty on the capture cross section by a factor of 10 to 15 . Therefore, an accurate measurement of capture cross section requires a precise knowledge of the IC efficiency. 

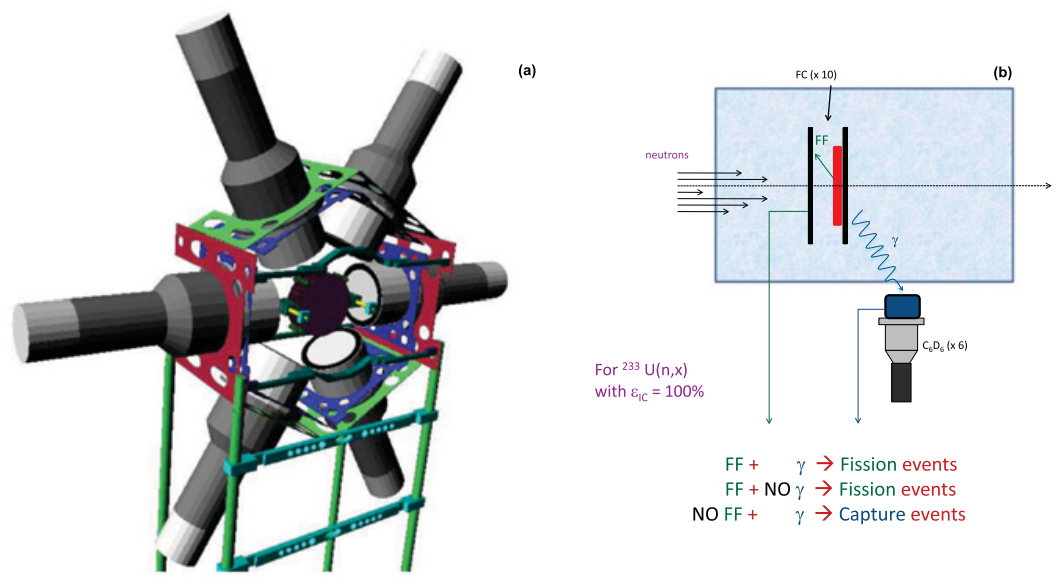

Figure 1. (a) Experimental set-up: six $\mathrm{C}_{6} \mathrm{D}_{6}$ detectors surrounding the IC containing the ${ }^{233} \mathrm{U}$ samples. (b) Capture to fission discrimination diagram.

(a)

(b)

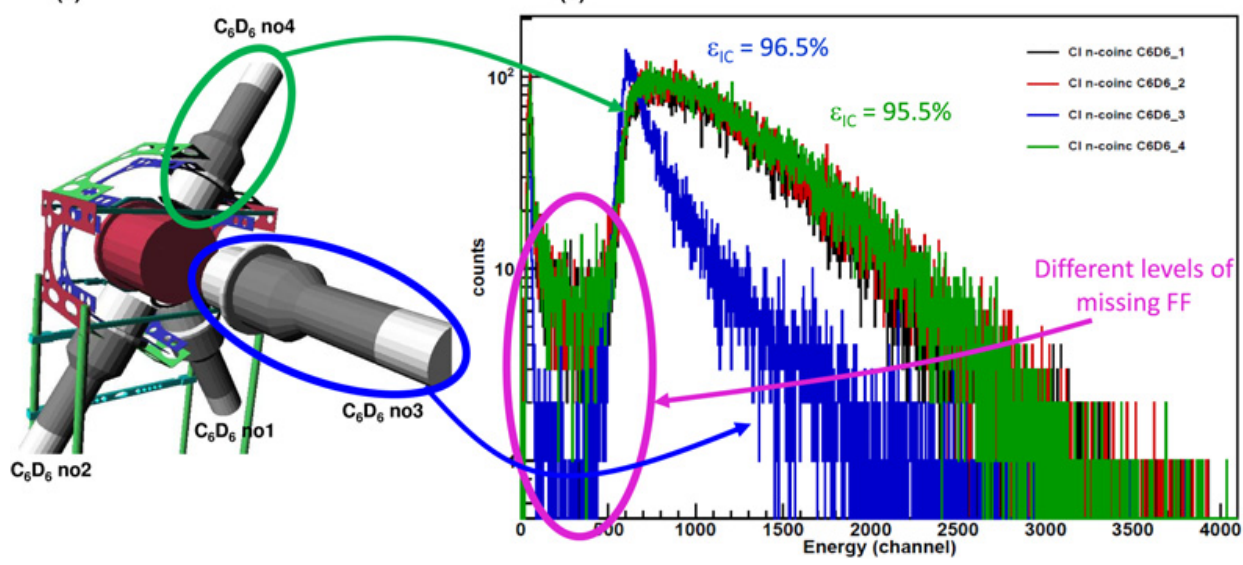

Figure 2. (a) Experimental set-up, three $\mathrm{C}_{6} \mathrm{D}_{6}$ detectors positioned around the IC and one $\mathrm{C}_{6} \mathrm{D}_{6}$ detector in front of the IC with the ${ }^{252} \mathrm{Cf}$ source. (b) Spectra of the IC in coincidence with the four $\mathrm{C}_{6} \mathrm{D}_{6}$ detectors.

\section{Results and discussion}

An experimental set-up has been designed at the CENBG. Complete description of the set-up is reported in [9]. The first measurement of the IC efficiency was done with a ${ }^{252} \mathrm{Cf}$ source, and by positioning three $\mathrm{C}_{6} \mathrm{D}_{6}$ detectors around the IC, and one in front of it as shown in Figure 2a. The pulse height spectra obtained with the IC in coincidence with each $\mathrm{C}_{6} \mathrm{D}_{6}$ are shown in Figure $2 \mathrm{~b}$.

The shape difference between the spectra can be explained by the kinematic effects between the FF and the neutrons. For instance, the $\mathrm{C}_{6} \mathrm{D}_{6}$ no1, $\mathrm{C}_{6} \mathrm{D}_{6}$ no2 and $\mathrm{C}_{6} \mathrm{D}_{6}$ no4 will detect most probably the neutrons coming from the FF emitted at razing angle. As these FF lose energy in the target they are detected in the valley. On the contrary the $\mathrm{C}_{6} \mathrm{D}_{6}$ no3, placed in front of the IC, will detect more probably the neutrons from the FF emitted on the forward direction which loose much less energy in the target. The associated spectrum is characterized by this selection of FF by generating a reduced amount of low-energy or high-energy events due to FF emitted at razing angles. 
The IC efficiency determined from this spectrum was found to be $96.5 \%$, whereas the efficiencies for the other spectra are $95.5 \%$. Measuring the real efficiency, with an uncertainty lower than $0.5 \%$, demands additional efforts to fully understand the method.

\section{Conclusions}

The capture cross section measurement of fissile nuclei with VETO method requires a very high and accurate FF detection efficiency. The prompt-neutron detection method is under investigation at CENBG in order to obtain the fission efficiency with a precision better than $0.5 \%$. First tests have shown that the kinematic effects play an important role in the determination of efficiency, as in spectrum obtained in coincidence with the detector placed in front of the IC.

\section{References}

[1] L. C. Leal et al., ORNL/TM-2000/372 (2001)

[2] A. Bidaud et al., Proc. of Nuclear Data for Sc. and Tech., Nice, France, 2, (2007) p. 919-923

[3] M. J. Cabell, M. Wilkins, J. Inorg. Nucl. Chem. 33, (1971) p. 3972

[4] F. D. Brooks, J. E. Joly, M. G. Schomberg, M. G. Sowerby, AERE, Harwell, Angleterre, React. Phys. Conf., 2, (1966) p. 193

[5] L. W. Weston, R. Gwin, S. de Saussure, Nucl. Sci. and Eng. 34, (1968) p. 1

[6] J. C. Hopkins et al., Nucl. Sci. and Eng. 12, (1962) p.169-177

[7] E. Berthoumieux et al., Proc. of Nuclear Data for Sc. and Tech., 2, (2007) p. 571-574

[8] A. Bensussan et J. M. Salome, Nucl. Inst. and Meth. 155, (1978) p. 11

[9] I. Companis et al., Proc. of Nucl. Reac. and Rel. Topics EPJ Web of Conference, 21, (2011) p. 1000 